\title{
Origin and Manipulation of Stable Vortex Ground States in Permalloy Nanotubes
}

\author{
Michael Zimmermann, ${ }^{\dagger}$ Thomas Norbert Gerhard Meier, ${ }^{\dagger}$ Florian Dirnberger, $^{\dagger}$ Attila Kákay, ${ }^{\ddagger}$ \\ Martin Decker, ${ }^{\dagger}$ Sebastian Wintz, ${ }^{\ddagger},{ }^{\S}$ Simone Finizio, ${ }^{\S}$ Elisabeth Josten, ${ }_{\dagger}^{\ddagger}$ Jörg Raabe, ${ }^{\S}$ Matthias Kronseder, ${ }^{\dagger}$ \\ Dominique Bougeard, ${ }^{\dagger}$ Jürgen Lindner, ${ }^{\dagger}$ and Christian Horst Back ${ }^{*} \dagger$ \\ ${ }^{\dagger}$ Physics Department, Universität Regensburg, Universitätsstraße 31, D-93053 Regensburg, Germany \\ ${ }^{\ddagger}$ Helmholtz-Zentrum, Dresden Rossendorf, Institute of Ion Beam Physics and Material Research, Bautzner Landstraße 400, 01328 \\ Dresden, Germany \\ ${ }^{\S}$ Paul Scherrer Institut, 5232 Villigen, Switzerland
}

ABSTRACT: We present a detailed study on the static magnetic properties of individual permalloy nanotubes (NTs) with hexagonal cross-sections. Anisotropic magnetoresistance (AMR) measurements and scanning transmission X-ray microscopy (STXM) are used to investigate their magnetic ground states and its stability. We find that the magnetization in zero applied magnetic field is in a very stable vortex state. Its origin is attributed to a strong growth-induced anisotropy with easy axis perpendicular to the long axis of the tubes. AMR

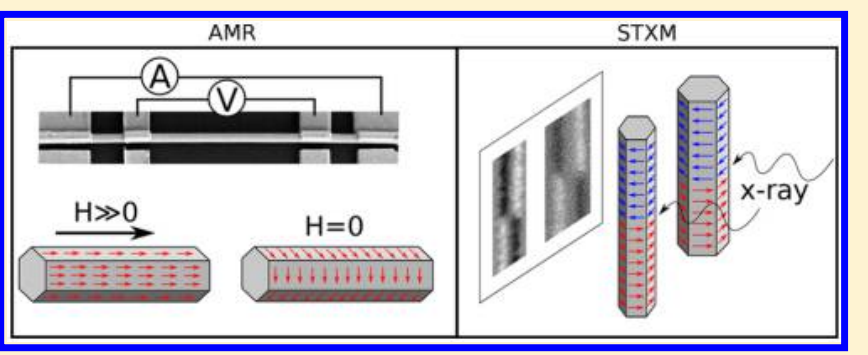
measurements of individual NTs in combination with micromagnetic simulations allow the determination of the magnitude of the growth-induced anisotropy for different types of NT coatings. We show that the strength of the anisotropy can be controlled by introducing a buffer layer underneath the magnetic layer. The magnetic ground states depend on the external magnetic field history and are directly imaged using STXM. Stable vortex domains can be introduced by external magnetic fields and can be erased by radio-frequency magnetic fields applied at the center of the tubes via a strip line antenna.

KEYWORDS: Nanotube, anisotropic magnetoresistance, permalloy, vortex, ground state

M agnetic nanoconduits have been proposed as future high-density memory elements. ${ }^{1}$ In such devices information can be encoded by writing domain walls (DWs) in a track. The DWs can be efficiently moved by spin-polarized currents, spin-orbit torques, or both. ${ }^{2,3}$ Ideally, a future domain wall memory device should allow for densely packed arrays, which requires stray field free states. ${ }^{4}$ Furthermore, the speed at which domain walls can be moved along a nanotrack determines the performance of such a device. A ferromagnetic nanotube (NT), with its lack of magnetic core, is a promising candidate for meeting both requirements. A variety of stable configurations for NTs are conceivable at zero external magnetic field, including a global vortex state (VS) in which the magnetization circulates around the circumference of the tube, a uniform magnetized state (US) in which the magnetization points along the long axis of the tube, an onion state (OS), and mixtures between these cases. ${ }^{5}$ These states are illustrated in Figure 1a. The metastable OS can only be obtained after the application of an external magnetic field perpendicular to the tube axis. Note that because the VS is a flux closed state, it enables the close packing of nanowires without magnetic cross-talk via stray fields. Furthermore, it has recently been predicted by micromagnetic simulations that DWs in magnetic NTs can be moved at extremely high speeds. ${ }^{6,7}$ Because the DWs introduced above and in particular their velocities are very different, the knowledge of the actual magnetic ground state and its control are key prerequisites for a successful implementation of magnetic NTs in modern storage technology.

Earlier attempts to determine the magnetic properties of NTs have mostly been conducted on large ensembles ${ }^{8-10}$ due to the small single magnetic moment of individual tubes. This, however, prevents an accurate characterization because ensembles of NTs exhibit a distribution in size, shape, and orientation depending strongly on the growth density. Therefore, the tube's individual magnetic properties may differ from those of the ensemble. Consequently, the investigation of single NTs with different ferromagnetic coatings came into the focus of research. ${ }^{11-15}$

While anisotropic magnetoresistance (AMR) measurements on single NTs have been performed, ${ }^{14-16}$ it remains challenging to draw conclusions from AMR data alone to the magnetic ground state. One reason may lie in a typically rough surface that makes it difficult to compare the experimental data

Received: December 12, 2017

Revised: March 28, 2018

Published: April 5, 2018 


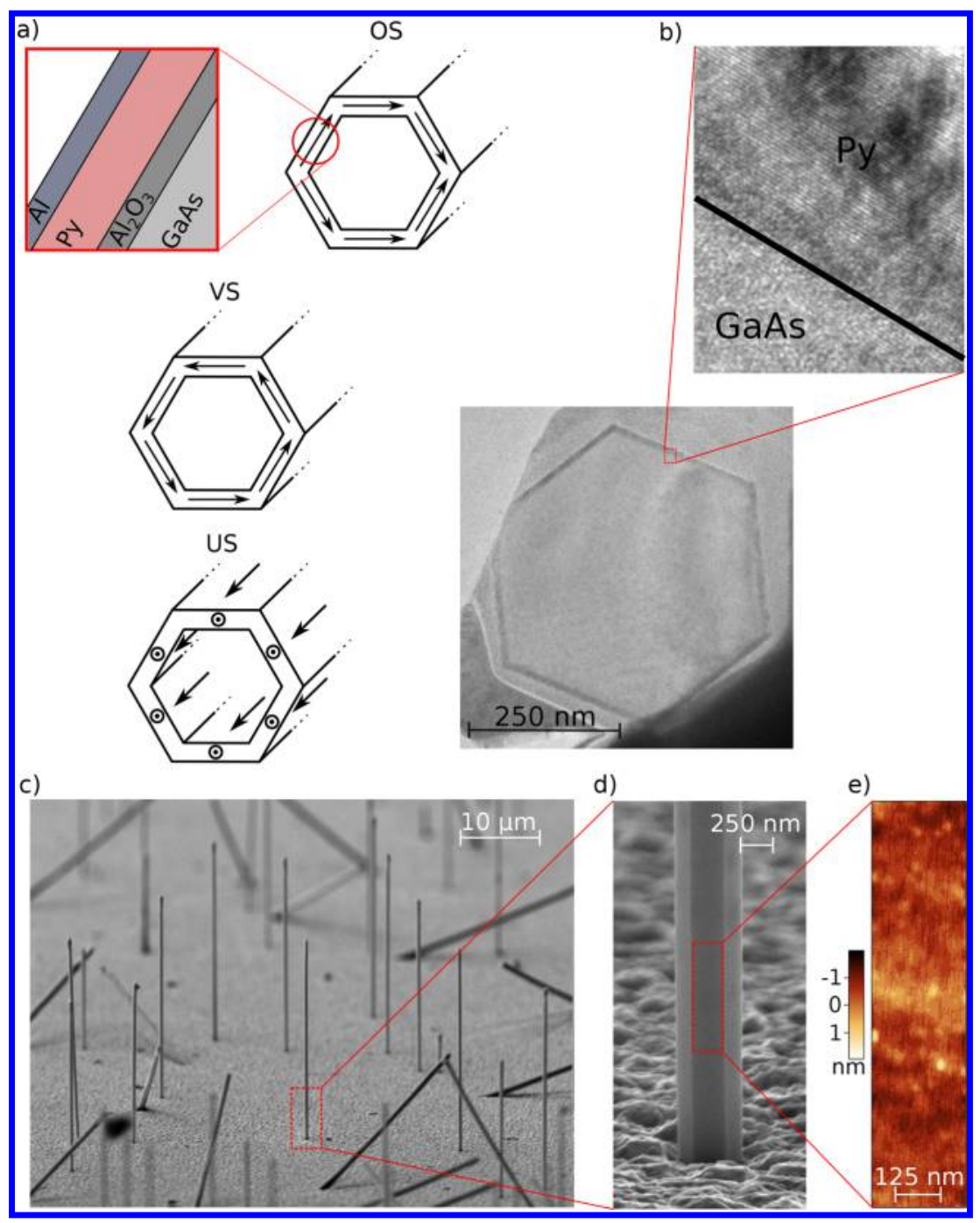

Figure 1. (a) Illustration of different states found in magnetic NTs and sketch of the layers deposited on the GaAs core. The thicknesses of the different layers for the samples are specified in Table 1. While the vortex state (VS) and the uniform state (US) may appear as magnetic ground states, the onion state (OS) is metastable and can only be entered after the application of an external magnetic field. (b) Cross-sectional TEM image of sample s1. The inset shows an enlargement of the border between GaAs and Py. The crystalline structure of Py can be identified. (c) SEM image of the free-standing, low-density NTs that are individually selected for the experiments. (d) Hexagonally shaped NTs exhibiting a very smooth surface after metal-layer deposition. (e) AFM images of the facets reveals a root mean squared roughness of $R_{\mathrm{rms}}=0.4 \mathrm{~nm}$.

with micromagnetic models of idealized tubes. In this Letter, we investigate nearly perfectly grown NTs in which the tubes and the magnetic coating have been grown in an ultra-high vacuum at very low base pressures. In addition, we exclusively process single NTs by using a dual-beam scanning electron microscope (SEM/FIB). On these grounds, we are able to avoid tapering effects appearing for densely grown NTs, guaranteeing a smooth, homogeneous magnetic shell.

For the analysis of individual NTs, two different techniques are applied. First, an AMR setup is used in which magnetic fields up to $2 \mathrm{~T}$ can be applied. The resistance change as a function of applied field gives access to the underlying magnetic ground state. A comparison of different field orientations, parallel and perpendicular to the tube's long axis, allows us to draw conclusions on the magnetic state in zero applied field. As a second tool, scanning transmission X-ray microscopy (STXM) at the $\mathrm{L}_{3}$-edge of iron $(\mathrm{Fe})$ is used to directly visualize the magnetic state by making use of X-ray magnetic circular dichroism $^{17}$ (XMCD) as contrast mechanism.
We find very stable vortex states, independent of the length of the tubes, indicating a different origin for the fluxed closed VS than the reduction of stray fields. ${ }^{3}$ Careful analysis of the AMR data and comparison with micromagnetic simulations allow the determination of the strength of the magnetic anisotropy for individual NTs. Using $\mathrm{Al}_{2} \mathrm{O}_{3}$ buffer layers, the anisotropy can be tuned such that the VS is less stable to externally applied magnetic fields. This ultimately leads to the conclusion that the VS is favored by a tunable growth induced azimuthal anisotropy, which, in turn, can be understood as a result of a shadowing effect occurring during growth at oblique angles. A growth-induced anisotropy has also been addressed in refs 15 and 18 as a possibility for the interpretation of the experimental findings.

Sample Design. In this study, NTs, consisting of nonmagnetic single crystalline GaAs cores and permalloy $\left(\mathrm{Ni}_{80} \mathrm{Fe}_{20}\right.$, Py $)$ shells, are chosen as objects of investigation. This choice is based on the characteristic low magnetic anisotropy of Py thin films, even on well-prepared singlecrystalline substrates. The NTs are fabricated in the following 


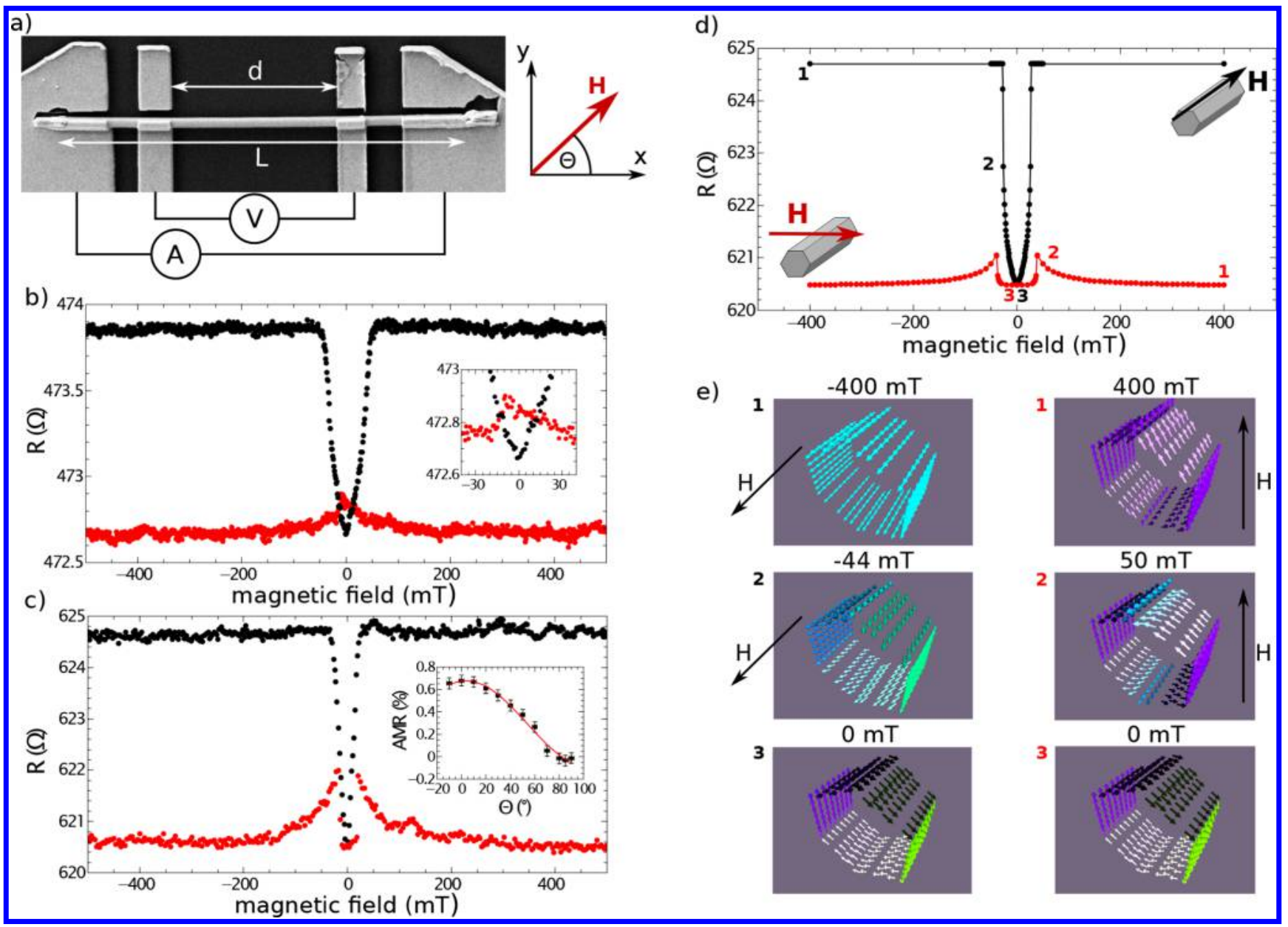

Figure 2. AMR measurements on individual NTs. (a) SEM image of a NT contacted by Au leads in a four-point configuration. The coordinate system describes the direction of the externally applied field $\mathbf{H}$. The magnetic field was swept from positive to negative values for all measurements. A linear background was subtracted from all obtained curves. Measurements for sample s1 and s2.1 are shown in panels b and c, respectively. The red (black) curves are obtained for $\Theta=90^{\circ}\left(0^{\circ}\right)$. (b) The inset shows a zoomed range of -40 to $40 \mathrm{mT}$. The inset in panel c shows the normalized AMR signal in the saturated state $\left(\mu_{0} H=1 \mathrm{~T}\right)$ as a function of the angle $\Theta$. (d) Simulation of the AMR experiment with parameters according to those of sample s2.1. (e) Images at selected field values according to the simulation shown in panel $\mathrm{d}$. The cross-sectional images of the NT display the magnetization for the given values and directions of $\mathbf{H}$.

two steps. First, GaAs rods are grown on oxidized $\mathrm{Si}(111)$ wafers via molecular-beam epitaxy (MBE) in a III-V MBE using Ga droplets as catalysts. ${ }^{9,19}$ After in situ transfer to a metal MBE in a pressure better than $1 \times 10^{-10} \mathrm{mbar}$, the coating layers $\left(\mathrm{Al}_{2} \mathrm{O}_{3} / \mathrm{Py} / \mathrm{Al}\right.$ or $\left.\mathrm{Py} / \mathrm{Al}\right)$ are deposited at pressures around $1 \times 10^{-10} \mathrm{mbar}$ (base pressure of $5 \times 10^{-11}$ mbar); see the left panel of Figure 1a. The $\mathrm{Al}_{2} \mathrm{O}_{3}$ layer is important for controlling the magnetic ground state and is, if deposited, $1 \mathrm{~nm}$ thick. The aluminum (Al) capping layer is 3 $\mathrm{nm}$ thick for all samples. The angle between the NTs axis and the evaporation direction of the Py source is $28^{\circ}$. During evaporation, the sample rotates at $10 \mathrm{rpm}$, producing a homogeneously thick coating on the NT. Figure $1 \mathrm{~b}-\mathrm{e}$ show a sample after growth. Figure $1 \mathrm{~b}$ shows a cross-section transmission electron microscopy (TEM) image of sample s1. The inset displays a magnified image of the region between GaAs and Py where the crystalline structure of Py can be identified. The SEM images in Figure 1c,d were taken at a grazing angle of roughly $85^{\circ}$ with respect to the substrate normal. The NT density is very low with approximately 1000 $\mathrm{NTs} / \mathrm{mm}^{2}$. While most of the tubes grow perpendicularly to the [111] surface normal, oblique NTs may occur for [001] and [110] crystalline directions. By means of atomic force microscopy (AFM) the root mean squared (rms) surface roughness on the side facets of a single NT is determined to be $0.4 \mathrm{~nm}$ (Figure 1e).

The hexagonally shaped NTs have a $15 \mathrm{~nm}$ thick magnetic shell with side facet normal vectors oriented along the [110] directions. The vertex-to-vertex diameter of the selected tubes is $500 \mathrm{~nm}$ and the tube's lengths range from 10 to $30 \mu \mathrm{m}$. To guarantee a homogeneous Py coating, a single NT is selected from an area where no other tubes are in its vicinity (compare this to Figure 1c). Hence, tapering effects due to shadowing during growth are completely avoided. Using a gas-injection system (GIS), the chosen NT is fixed with platinum on the tip of a nanomanipulator. A focused ion beam (FIB) then detaches the NT from the substrate, and it is placed elsewhere on an oxidized silicon sample. Finally specific lengths and straight ends are created using precise FIB cuts. The obtained individual NT can, for instance, be contacted with gold stripes (Figure 2a) via electron beam lithography for transport measurements.

The exclusion of tapering, straight tube ends and low surface roughness provide samples closely resembling model samples used in simulations. Therefore, a direct comparison of the 
results from experimental measurements and simulations becomes possible. For the simulations, standard Py material parameters are used: $M_{\mathrm{S}}=800 \mathrm{kA} / \mathrm{m}$ and $A_{\mathrm{ex}}=13 \times 10^{-12} \mathrm{~J} /$ $\mathrm{m}$. Furthermore, we simulated a maximal cell size of $4 \mathrm{~nm}$, which is below the exchange length of $\mathrm{Py}: l_{\mathrm{ex}} \approx 5.7 \mathrm{~nm}$. For most simulations periodic boundary conditions are applied to a cross-sectional cut of the nanotube. This treatment is justified due to the high anisotropies encountered in our samples, leading to a homogeneous magnetic profile along the tubes length. In the simulations the AMR response is calculated from the cosine square of the local angle between magnetization and current. Hence, we do not account for the dependence of the AMR on out-of-plane or in-plane magnetization. ${ }^{20}$ Because the maximal simulated out-of-plane tilting angle is only $18.7^{\circ}$, this effect is negligible.

AMR Measurements. This section presents AMR measurements conducted on three different NTs (s1, s2.1, and s2.2). Their properties and the obtained results are summarized in Table 1 . The resistance $R$ is measured as a function of external

Table 1. Overview of the Properties of the Three Different NTs (s1, s2.1, and s2.2) Investigated via $\mathrm{AMR}^{a}$

\begin{tabular}{llll}
\multicolumn{1}{c}{ name } & \multicolumn{1}{c}{$\mathrm{s1}$} & \multicolumn{1}{c}{$\mathrm{s} 2.1$} & \multicolumn{1}{c}{$\mathrm{s} 2.2$} \\
layer & $\mathrm{Py} / \mathrm{Al}$ & $\mathrm{Al}_{2} \mathrm{O}_{3} / \mathrm{Py} / \mathrm{Al}$ & $\mathrm{Al}_{2} \mathrm{O}_{3} / \mathrm{Py} / \mathrm{Al}$ \\
$L(\mu \mathrm{m})$ & 20 & 20 & 30 \\
$d(\mu \mathrm{m})$ & 9 & 9 & 15 \\
$\mathrm{AMR}(\%)$ & $0.25 \pm 0.03$ & $0.68 \pm 0.05$ & $0.74 \pm 0.05$ \\
$R_{0}(\Omega)$ & $472.5 \pm 0.2$ & $620.5 \pm 0.2$ & $700 \pm 0.4$ \\
$\mu_{0} H_{\text {sat, } \|}(\mathrm{mT})$ & $53 \pm 3$ & $30 \pm 3$ & $30 \pm 3$ \\
type & 4-point & 2-point & 4-point \\
\hline
\end{tabular}

${ }^{a} H_{\text {sat, } \|}$ is the external magnetic field necessary to align the magnetization $\mathbf{M}$ parallel to the tube's long axis. $R_{0}$ is the resistance at zero applied field, $d$ is the distance between the voltage contacts, and $L$ is the length of the tube.

magnetic field $\mathbf{H}$ for various directions indicated by the angle $\Theta$ in Figure 2(a). The applied current $I$ was varied between 1 and $10 \mu \mathrm{A}$. Due to the insulating $\mathrm{Al}_{2} \mathrm{O}_{3}$ buffer layer of s2.1 and s2.2, a higher current flows in the Py-layer than for s1, where a significant portion $(\sim 24 \%)$ flows through the semiconducting GaAs core. This unwanted current-flow in the semiconductor reduces the maximum AMR ratio defined as AMR $=\left(R_{\|}-\right.$ $\left.R_{\perp}\right) / R_{\perp}$ by roughly a factor of 3 (compare this to Table 1 ) but does not influence the interpretation of the data. Furthermore, the signal-to-noise ratio deteriorates, which can only be partially compensated by enhancing the current. Typically, $I=2(5) \mu \mathrm{A}$ have been applied for $\mathrm{s} 2.1 / \mathrm{s} 2.2$ (s1).

Panels $b$ and $c$ in Figure 2 display resistance measurements for $\mathrm{s} 1$ and s2.1, respectively. The black curves, obtained for $\Theta=$ 0 , immediately reveal that the remaining state can not be a saturated state pointing along the tubes axis because the zerofield resistance differs drastically from the high-field resistance. Because the onion state can only be entered after application of an external field perpendicular to the tube axis the ground state is a VS. Given that the magnetization in the VS is perpendicular to the tubes long axis, the resistance is decreasing while approaching zero external field. For s1 (s2.1), an applied field of $H_{\text {sat }, \|}=53 \mathrm{mT}(30 \mathrm{mT})$ is necessary to rotate the magnetic moments completely into the direction parallel to the tube's long axis, thus identified as the hard axis. The corresponding energy density difference $\Delta \epsilon$ between US and VS can be calculated using the relation $\mu_{0} \cdot H_{\text {sat, } \|}=\frac{2 \Delta \epsilon}{M_{S}}$, where $\mu_{0} \mathrm{M}_{\mathrm{S}}=1 \mathrm{~T}$ is the saturation magnetization of Py. Hence, $\Delta \epsilon=21200 \mathrm{~J} / \mathrm{m}^{3}$ for s1 and $\Delta \epsilon=13200 \mathrm{~J} / \mathrm{m}^{3}$ for s2.1 and s2.2. The strength of the anisotropy is calculated in the following. The total energy density difference between the US and the VS is given by:

$$
\begin{aligned}
& \Delta \epsilon=\Delta \epsilon_{\mathrm{ex}}+\Delta \epsilon_{\mathrm{ani}}+\Delta \epsilon_{\mathrm{demag}} \\
& \Delta \epsilon=\left(0-\epsilon_{\mathrm{ex}, \mathrm{VS}}\right)+\left(\epsilon_{\mathrm{ani}, \mathrm{US}}-0\right)+\left(\epsilon_{\mathrm{dem}, \mathrm{US}}-0\right)
\end{aligned}
$$

comprising the exchange energy density $\epsilon_{\mathrm{ex}, \mathrm{VS}}$, induced by the curvature of the six edges in the VS, the anisotropy energy density $\epsilon_{\text {ani,US, }}$ and the demagnetizing energy density $\epsilon_{\text {dem,US }}$ for the case of a NT in the US. The anisotropy is assumed to be of uniaxial character and can be described by $\epsilon_{\text {ani,Us }}=-K_{2} \cos _{2} \Theta$, where $K_{2}$ is the uniaxial anisotropy constant and the angle $\Theta$ is introduced in Figure 2a. The last nonzero term in eq 2 can be estimated by approximating the NT by a general ellipsoid, yielding: ${ }^{21}$

$$
\epsilon_{\mathrm{dem}, \mathrm{US}}=\frac{1}{2} \mu_{0} M_{\mathrm{S}}^{2} \frac{t}{t+L}=300 \frac{\mathrm{J}}{\mathrm{m}^{3}}
$$

where $t=15 \mathrm{~nm}$ is the thickness of the Py coating and $L=20$ $\mu \mathrm{m}$ is the length of the NT. The exchange contribution to the energy density of the VS $\epsilon_{\text {ex,VS }}$ is estimated using micromagnetic simulations conducted with $\mathrm{K}_{2}^{s 1}=-21000 \mathrm{~J} / \mathrm{m}^{3}$ and $\mathrm{K}_{2}^{s 2}=$ $-13000 \mathrm{~J} / \mathrm{m}^{3}$. The latter is exemplary shown in Figure $2 \mathrm{~d}$. From these simulations, the values $H_{\text {sat, } \|, \text { sim }}^{\text {s1 }}=48 \mathrm{mT}$ and $H_{\text {sat }, \| \text {,sim }}^{s 2}=28 \mathrm{mT}$ are obtained. Applying these values and the result of eq 3 to eq 2 yields $\epsilon_{\mathrm{ex}, \mathrm{VS}, \mathrm{sim}}^{s 1}=2100 \mathrm{~J} / \mathrm{m}^{3}$ and $\epsilon_{\mathrm{ex}, \mathrm{VS}, \mathrm{sim}}^{s 2}=$ $2100 \mathrm{~J} / \mathrm{m}^{3}$. As expected, the exchange energy density does not change for different anisotropies and can be used to obtain $K_{2}$ from the measurements. Using the values for $\mu_{0} H_{\text {sat, } \|}$ listed in Table 1 and applying $\epsilon_{\mathrm{ex}, \mathrm{VS}}=2100 \mathrm{~J} / \mathrm{m}^{3}$ and $\epsilon_{\mathrm{dem}, \mathrm{US}}=300 \mathrm{~J} / \mathrm{m}^{3}$ to eq 2 finally gives $K_{2}^{s 1}=-23000 \mathrm{~J} / \mathrm{m}^{3}$ and $K_{2}^{s 2}=-15000 \mathrm{~J} /$ $\mathrm{m}^{3}$ for $\mathrm{s} 1$ and $\mathrm{s} 2$, respectively. Hence, the $1 \mathrm{~nm}$ thick $\mathrm{Al}_{2} \mathrm{O}_{3}$ layer reduces the anisotropy by a factor of 1.5 .

A typical angular dependence of the AMR in the saturated state at $1 \mathrm{~T}$ is shown exemplarily for $\mathrm{s} 2$ in the inset of Figure 2c. The normalized AMR signal shows a maximum (minimum) corresponding to a parallel (perpendicular) orientation of current and external field, respectively. The data follows the well-known $\cos ^{2} \Theta$ dependence and the corresponding fit is shown as red line.

A major difference between sample s1 and s2.1 is observed in the behavior of the AMR when approaching zero field with $\Theta=$ $90^{\circ}$. The field sweep direction is always from positive to negative fields. s2.1 switches into the vortex ground state for field values below $15 \mathrm{mT}$, visible in the AMR measurements as an abrupt resistance drop. In contrast, s1 remains in a metastable onion-like state with a larger resistance at zero applied field. The inset in Figure $2 b$ shows the field regions between -40 and $40 \mathrm{mT}$. For s1, the original onion state is left at $-10 \mathrm{mT}$, possibly for another onion-like state with orientation switched by $180^{\circ}$. Simulations conducted with the parameters corresponding to s1 and s2.1 always lead to the vortex state at zero field. Panels $\mathrm{d}$ and e of Figure 2 show the simulation according to the parameters of s2.1. In the simulations, the vortex state is reached for an applied magnetic field twice as large than observed experimentally. The onionlike metastable state of $\mathrm{s} 1$ at zero field may be attributed to pinning effects that were not included in the simulations. These seem to be pronounced if the Py layer is directly grown on GaAs. 


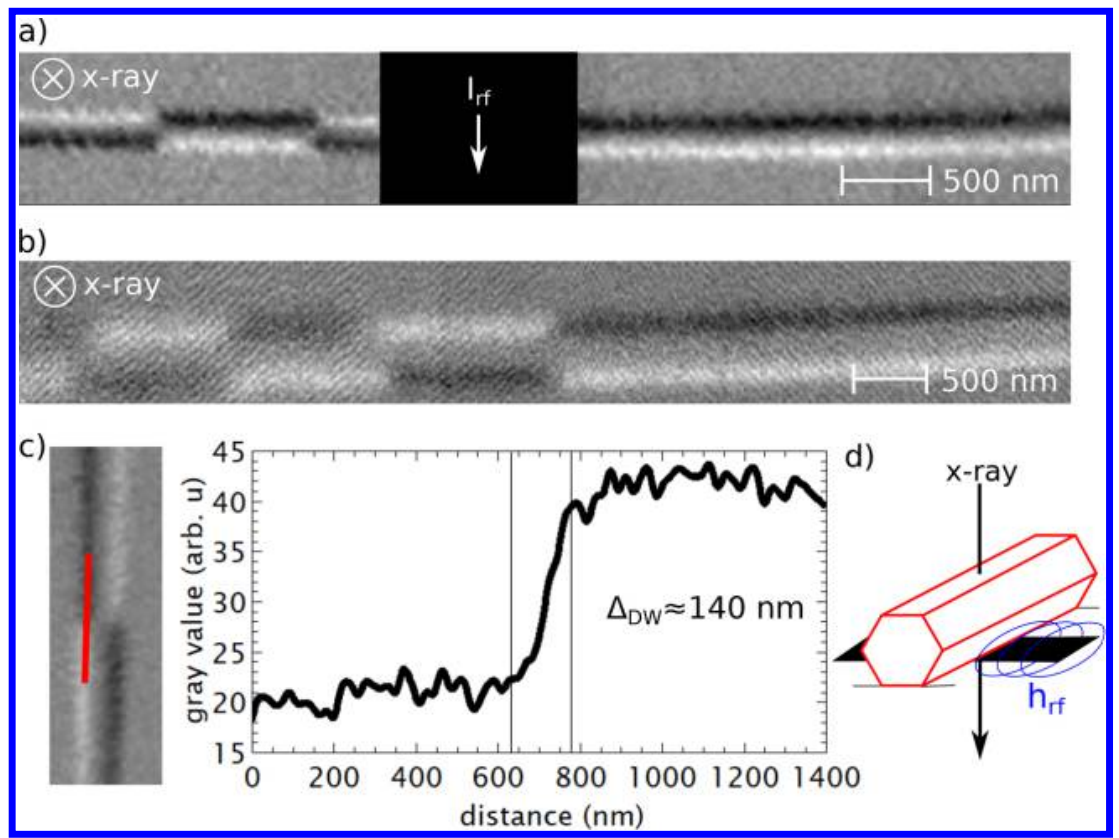

Figure 3. XMCD images obtained with STXM at zero applied magnetic field. Black and white contrast corresponds to a magnetization component pointing parallel and antiparallel to the X-ray beam direction, which is illustrated in panel d. (a) A $210 \mathrm{~nm}$ diameter tube placed on top of a $1 \mu \mathrm{m}$ wide antenna to be able to apply rf-excitation fields. (b) A $500 \mathrm{~nm}$ diameter tube. Domains are clearly visible for both tube types. (c) An enlarged image section of the $210 \mathrm{~nm}$ tube. A line scan is shown along the red line to estimate an upper bound of the domain wall width: $\Delta_{\mathrm{DW}}=140 \mathrm{~nm}$. The lateral size of the X-ray beam is approximately $40 \mathrm{~nm}$. (d) Illustration of the X-ray beam direction with respect to the NT facets orientation. The excitation field $h_{\mathrm{rf}}$ is generated by the strip antenna.

The remainder of this section discusses the origin and the tunability of the large anisotropy $K_{2}$, described above. To account for crystalline anisotropies, a Py full film sample grown on $\mathrm{GaAs}(110)$ was analyzed via conventional absorption ferromagnetic resonance. For this sample, we find a uniaxial in-plane anisotropy of $K_{2}=3000 \mathrm{~J} / \mathrm{m}^{3}$. Hence, crystalline anisotropy itself can not be responsible for the large anisotropies found in the NTs, especially because the orientation of the anisotropy does not coincide with the NTs circumference, which would be necessary to favor the VS.

The role of the demagnetizing field on the ground state arising from the different NT length is investigated in the following. Samples s2.1 and s2.2 were both picked from the same wafer; thus, their only difference is their length $(L=20$ $\mu \mathrm{m}$ for $\mathrm{s} 2.1$ and $L=30 \mu \mathrm{m}$ for s2.2). When the external field is applied at $\Theta=0$, a saturation field $H_{\text {sat }, \|}$ is necessary to drag the magnetization out of the vortex state into the US. The results listed in Table 1 reveal no difference for both lengths, confirming the negligible contribution of the demagnetizing energy for the investigated NT-parameters and lengths. Applying eq 3, the difference for $L=20 \mu \mathrm{m}$ and $L=30 \mu \mathrm{m}$ is approximated to be only $0.25 \mathrm{mT}$. This result can be confirmed by micromagnetic simulations assuming no anisotropy: a NT with magnetic parameters corresponding to s2.1 exhibits a ground-state magnetization pointing along the tube's long axis, with edge vortex domains on each side of roughly 1 $\mu \mathrm{m}$ in length. In good approximation, these edge domains remain the same size and independent of the NT length. Similar behavior has been found in earlier simulations for cylindrical NTs. ${ }^{5}$

The origin of the enormous anisotropy can be explained by a self-shadowing effect, which occurs during the growth process. This effect is well-known and has been studied in several earlier works on full-film samples. ${ }^{22-25}$ However, it has not been considered in the context of MBE-grown magnetic NT coatings. A prerequisite for its occurrence is a small angle between long axis of the tubes and the Py evaporation beam direction, referred to as $\zeta$. The "surface" in the case of a NT is given by the facets. For our setup, $\zeta=28^{\circ}$ for all facets. During the initial stages of the Py layer formation, small crystallites are randomly distributed across the facets. These crystallites act further as growth nuclei, while the facet region behind them is deprived of Py deposition leading to a shadowing effect. At some point, the crystallites get large enough to start merging, creating crystalline chains whose long axes tend to be perpendicular to the beam direction. ${ }^{23}$ In the case of NTs, this axis therefore circles the circumference, creating a growthinduced anisotropy that favors the vortex ground state.

Knowledge of the origin of the growth-induced anisotropy is essential to modifying the NTs magnetic properties, such as the stability of the ground state at zero applied field. A comparison of $\mathrm{s} 1$ and s2.1, whose only difference is the insulating buffer layer $\mathrm{Al}_{2} \mathrm{O}_{3}$, shows a difference in $H_{\text {sat, } \|}$ of $23 \mathrm{mT}$, which is directly linked to an anisotropy difference of $\Delta K_{2}=8000 \mathrm{~J} / \mathrm{m}^{3}$. The $1 \mathrm{~nm}$ thick buffer layer creates a slightly rougher surface and influences the crystalline growth of Py due to its amorphous structure. Without the buffer layer, the crystalline structure of Py is adapted directly from the GaAs. For a $1 \mathrm{~nm}$ thick $\mathrm{Al}_{2} \mathrm{O}_{3}$ layer, the crystalline growth on the facets is influenced enough to reduce the induced anisotropy by a factor of 1.5 (difference between sample s1 and s2). In other reports, ${ }^{12,13}$ the GaAs rods are transferred to a MBE chamber without in situ connection. Consequently, the surface of the GaAs oxidizes and the reported results suggest almost no growth induced anisotropy. We expect similar findings for thicker layers of $\mathrm{Al}_{2} \mathrm{O}_{3}$.

Another approach to changing the magnetic ground state of the NT would be a variation of the growth conditions. For 
instance, different angles $(\zeta)$ can change the growth-induced anisotropy. ${ }^{22-25}$ While $\zeta=90^{\circ}$ would result in zero growthinduced anisotropy, as it is known for Py thin films, smaller angles than $90^{\circ}$ induce an anisotropy perpendicular to the Py atoms beam direction, with a maximum anisotropy at around $40^{\circ}{ }^{22,23,25}$ This anisotropy favors the vortex state in the case of nanotubes. Unfortunately, changing $\zeta$ is impossible in most growth chambers because it requires a repositioning of the evaporation source. Hence, as a more-comfortable parameter, the growth temperature can be exploited to tune the growthinduced anisotropy and thus the ground state in NTs. Increasing the mobility of the metal atoms on the facets reduces the anisotropy because atoms are able to diffuse into the shadowed regions during the initial states of growth and thus influence the crystalline chains. This effect has already been investigated for flat Py films. ${ }^{23}$

STXM Measurements. This section presents the scanning transmission X-ray microscopy measurements conducted at the PolLux $^{26}$ (X07DA) end-station of the Swiss Light Source synchrotron at the Paul Scherrer Institute. To visualize the magnetization of the $\mathrm{NTs}^{27,28}$ images with left and right circularly polarized light are taken, respectively. The absorption spectra are obtained at the $\mathrm{L}_{3}$-edge of iron $(709.8 \mathrm{eV})$. Their difference spectrum is obtained by calculating the XMCD asymmetry, given by $I_{\mathrm{XMCD}}=\left(I_{\text {left }}-I_{\text {right }}\right) /\left(I_{\text {left }}+I_{\text {right }}\right)$. The XMCD method is only sensitive to the component of the magnetization collinear to the X-ray beam ( $\mathbf{M} \| \mathrm{X}$-ray beam).

A pair of different NTs are discussed in the following material. A $210 \mathrm{~nm}$ diameter tube with a $30 \mathrm{~nm}$ thick Py shell and a $500 \mathrm{~nm}$ diameter tube with a $15 \mathrm{~nm}$ thick Py shell, both capped with $3 \mathrm{~nm} \mathrm{Al}$, are used. The samples are shown in panels a and b of Figure 3, respectively. Both samples are grown without buffer layers, meaning that the Py layer is in direct contact with the GaAs core (compare this to sample s1 in Table 1). The samples are positioned such that the top facet normal is parallel to the X-ray beam (compare this to Figure 3d). Because STXM imaging is a transmission technique, in the case of a tube in the VS, the magnetic signal of upper and lower facets average to zero, while the oblique facets accumulate their signals. The obtained images in Figure 3 clearly visualize the vortex state described in the first section.

The images shown in panels $a$ and $b$ of Figure 3 were obtained after application and removal of a magnetic field of $150 \mathrm{mT}$ perpendicular to the NT. Initially, the NTs exhibited a global VS for both types of tubes. Our previously displayed AMR findings are therefore nicely confirmed using a complementary technique. However, we observe that after removing the external field the tubes decay into sections of vortex domains with opposite circulation around the NT. A line scan across a domain wall is shown in Figure $3 \mathrm{c}$. The lateral size of the X-ray beam is approximately $40 \mathrm{~nm}$ in these experiments, i.e., the measured domain wall width of $\Delta_{\mathrm{DW}}=\pi l=140 \mathrm{~nm}$ represents an upper limit. To estimate the actual value of the domain wall parameter $l$, we use micromagnetic simulations, applying the sample parameters of $\mathrm{s} 1\left(K_{2}=23000 \mathrm{~J} / \mathrm{m}^{3}\right)$. In the simulation a DW is forced into a NT by starting with two opposing vortex domains. After the relaxation process, the DW width is determined to be $\Delta_{\mathrm{DW}} \approx 70 \mathrm{~nm}$ for the $500 \mathrm{~nm}$ as well as for the $250 \mathrm{~nm}$ diameter tube. $l$ can be used to estimate the number of domain walls necessary to account for the AMR signal in Figure $2 \mathrm{~b}$ if assuming a multi-domain state at zero applied field instead of a metastable onion state. The domain wall profile is expressed as $m_{x}(x)=\cosh ^{-1}(x / l)$, where $x$ is the direction parallel to the tubes axis. Because the AMR signal depends quadratically on $m_{x}$, the contribution of one single domain wall to the AMR signal is $\int_{-\infty}^{\infty} m_{x}(x)^{2} \mathrm{~d} x=2 l$. When using this approximation, there would need to be more than 35 domains within the distance between the voltage contacts $(d=$ $9 \mu \mathrm{m}$ ) to account for the increase in resistance at zero applied field (red curve in Figure 2b). Because we never encountered such a high density of domain walls, the onion state is still a reasonable explanation to account for the increase in resistance approaching zero field.

In an attempt to achieve a uniform magnetic state, both types of tubes are placed on a copper strip line to be able to apply rf magnetic fields. For Figure $3 b$, the antenna is on the far right (outside the image section). The application of $\mathrm{rf}$ excitation for some seconds, exceeding $4 \mathrm{dBm}$ throughput, leads to the removal of all initially created domains, leaving only a global vortex state (not shown). The power of $4 \mathrm{dBm}$ corresponds to a field of roughly $5 \mathrm{mT}$ directly at the facet in contact with the antenna (width of $1 \mu \mathrm{m}$ and thickness of $250 \mathrm{~nm}$ ). Domains are removed for all tested frequencies $(2-8 \mathrm{GHz})$. The exact process of domain wall removal is unresolved at the moment, but we speculate that the excitation of the magnetization with very large rf magnetic fields leads to the excitation of highamplitude spin waves, which push the domain walls toward the ends of the tubes, where they are annihilated. Because applications rely on reading and writing processes, this finding is of particular interest for further investigations.

Conclusions. In conclusion, anisotropic magnetoresistance and transmission X-ray microscopy measurements were conducted on individually selected Py NTs. A very stable vortex ground state was found for Py grown directly on the GaAs core. The large anisotropy necessary for the VS formation can be explained in terms of a growth induced effect, depending strongly on the growth conditions such as the angle between the NT axis and the thermal Py evaporation source. When using $1 \mathrm{~nm}$ thick $\mathrm{Al}_{2} \mathrm{O}_{3}$ buffer layers between $\mathrm{Py}$ and GaAs, the anisotropy can be reduced by a factor of 1.5. These findings offer the possibility to control the magnetic ground states of NTs during growth. The flux closed VS is particularly interesting for densely packed arrays of NTs because it greatly reduces magneto-static interactions. To corroborate our findings, the vortex ground state was directly visualized with $\mathrm{X}$-ray magnetic microscopy. Stable vortex domain walls can be introduced into the NT when external magnetic fields are applied perpendicular to the tube's axis for a short period of time. Surprisingly, these domains can be erased again when a strong local rf-magnetic field excitation is applied. Our findings may enable a broad range of further experiments concerning domain wall motion in magnetic NTs as well as applications using NTs as building blocks for future data-storage devices.

\section{AUTHOR INFORMATION}

\section{Corresponding Author}

*E-mail: christian.back@ur.de. Phone: +49-941943-2621. Fax: +49-941943-4544.

\section{ORCID $\odot$}

Michael Zimmermann: 0000-0002-1945-1237

\section{Notes}

The authors declare no competing financial interest. 


\section{ABBREVIATIONS}

NT, nanotube; AMR, anisotropic magnetoresistance; STXM, scanning transmission X-ray microscopy; DW, domain wall; VS, vortex state; US, uniform state; OS, onion state; SEM, scanning electron microscope; FIB, focused ion beam; XMCD, X-ray magnetic circular dichroism; $\mathrm{MBE}$, molecular-beam epitaxy; TEM, transmission electron microscopy; AFM, atomic force microscopy; GIS, gas-injection system

\section{REFERENCES}

(1) Parkin, S. S. P.; Hayashi, M.; Thomas, L. Magnetic Domain-Wall Racetrack Memory. Science 2008, 320, 190-194.

(2) Emori, S.; Bauer, U.; Ahn, S.-M.; Martínez, E.; Beach, G. S. D. Current-driven dynamics of chiral ferromagnetic domain walls. Nat. Mater. 2013, 12 (7), 611-616.

(3) Ryu, K.-S.; Thomas, L.; Yang, S.-H.; Parkin, S. Chiral spin torque at magnetic domain walls. Nat. Nanotechnol. 2013, 8, 527-533.

(4) Lin, W.-Y.; Ho, C.-C.; Hsu, W.-K. Large-scale production of ferromagnetic nanorings by a modified hole-mask colloidal lithography: Controlled creation of flux-closure vortex state. AIP Adv. 2016, 6, 025102 .

(5) Landeros, P.; Suarez, O. J.; Cuchillo, A.; Vargas, P. Equilibrium states and vortex domain wall nucleation in ferromagnetic nanotubes. Phvs. Rev. B: Condens. Matter Mater. Phvs. 2009, 79, 024404.

(6) Yan, M.; Andreas, C.; Kákay, A.; García-Sánchez, F.; Hertel, R. Fast domain wall dynamics in magnetic nanotubes: Suppression of Walker breakdown and Cherenkov-like spin wave emission. Appl. Phvs. Lett. 2011, 99, 122505.

(7) Otálora, J. A.; Yan, M.; Schultheiss, H.; Hertel, R.; Kákay, A. Curvature-Induced Asymmetric Spin-Wave Dispersion. Phvs. Rev. Lett. 2016, 117, 227203.

(8) Bachmann, J.; Jing; Knez, M.; Barth, S.; Shen, H.; Mathur, S.; Gösele, U.; Nielsch, K. Ordered Iron Oxide Nanotube Arrays of Controlled Geometry and Tunable Magnetism by Atomic Layer Deposition. I. Am. Chem. Soc. 2007, 129, 9554-9555.

(9) Rudolph, A.; Soda, M.; Kiessling, M.; Wojtowicz, T.; Schuh, D.; Wegscheider, W.; Zweck, J.; Back, C.; Reiger, E. Ferromagnetic GaAs/ GaMnAs Core-Shell Nanowires Grown by Molecular Beam Epitaxy. Nano Lett. 2009, 9, 3860-3866.

(10) Albrecht, O.; Zierold, R.; Allende, S.; Escrig, J.; Patzig, C.; Rauschenbach, B.; Nielsch, K.; Görlitz, D. Experimental evidence for an angular dependent transition of magnetization reversal modes in magnetic nanotubes. I. Appl. Phvs. 2011, 109, 093910.

(11) Gross, B.; Weber, D. P.; Rüffer, D.; Buchter, A.; Heimbach, F.; Fontcuberta i Morral, A.; Grundler, D.; Poggio, M. Dynamic cantilever magnetometry of individual $\mathrm{CoFeB}$ nanotubes. Phvs. Rev. B: Condens. Matter Mater. Phvs. 2016, 93, 064409.

(12) Buchter, A.; Wölbing, R.; Wyss, M.; Kieler, O. F.; Weimann, T.; Kohlmann, J.; Zorin, A. B.; Rüffer, D.; Matteini, F.; Tütüncüoglu, G.; et al. Magnetization reversal of an individual exchange-biased permalloy nanotube. Phys. Rev. B: Condens. Matter Mater. Phys. 2015, 92, 214432

(13) Wyss, M.; Mehlin, A.; Gross, B.; Buchter, A.; Farhan, A.; Buzzi, M.; Kleibert, A.; Tütüncüoglu, G.; Heimbach, F.; Fontcuberta i Morral, A.; Grundler, D.; Poggio, M. Imaging magnetic vortex configurations in ferromagnetic nanotubes. Phvs. Rev. B: Condens. Matter Mater. Phvs. 2017, 96, 024423.

(14) Rüffer, D.; Huber, R.; Berberich, P.; Albert, S.; Russo-Averchi, E.; Heiss, M.; Arbiol, J.; Fontcuberta i Morral, A.; Grundler, D. Magnetic states of an individual $\mathrm{Ni}$ nanotube probed by anisotropic magnetoresistance. Nanoscale 2012, 4, 4989-4995.

(15) Baumgaertl, K.; Heimbach, F.; Maendl, S.; Rueffer, D.; Fontcuberta I Morral, A.; Grundler, D. Magnetization reversal in individual $\mathrm{Py}$ and $\mathrm{CoFeB}$ nanotubes locally probed via anisotropic magnetoresistance and anomalous Nernst effect. Appl. Phvs. Lett. 2016, $108,132408$.

(16) Rüffer, D.; Slot, M.; Huber, R.; Schwarze, T.; Heimbach, F.; Tütüncüoglu, G.; Matteini, F.; Russo-Averchi, E.; Kovács, A.; Dunin-
Borkowski, R.; Zamani, R. R.; Morante, J. R.; Arbiol, J.; i Morral, A. F.; Grundler, D. Anisotropic magnetoresistance of individual $\mathrm{CoFeB}$ and $\mathrm{Ni}$ nanotubes with values of up to $1.4 \%$ at room temperature. $A P L$ Mater. 2014, 2, 076112.

(17) Schütz, G.; Wagner, W.; Wilhelm, W.; Kienle, P.; Zeller, R.; Frahm, R.; Materlik, G. Absorption of circularly polarized x-rays in iron. Phvs. Rev. Lett. 1987, 58, 737-740.

(18) Stano, M. et al. Imaging magnetic flux-closure domains and domain walls in electroless-deposited CoNiB nanotubes. 2017, arXiv:1704.06614v1. arXiv.org e-Print archive. https://arxiv.org/abs/ 1704.06614 (accessed 3/10/2018).

(19) Uccelli, E.; Arbiol, J.; Magen, C.; Krogstrup, P.; Russo-Averchi, E.; Heiss, M.; Mugny, G.; Morier-Genoud, F.; Nygård, J.; Morante, J. R; Fontcuberta i Morral, A. Three-Dimensional Multiple-Order Twinning of Self-Catalyzed GaAs Nanowires on Si Substrates. Nano Lett. 2011, 11, 3827-3832.

(20) Rijks, T. G. S. M.; Coehoorn, R.; de Jong, M. J. M.; de Jonge, W. $\mathrm{J}$. M. Semiclassical calculations of the anisotropic magnetoresistance of NiFe-based thin films, wires, and multilayers. Phvs. Rev. B: Condens. Matter Mater. Phys. 1995, 51, 283-291.

(21) Osborn, J. A. Demagnetizing Factors of the General Ellipsoid. Phys. Rev. 1945, 67, 351-357.

(22) Cohen, M. S. Anisotropy in Permalloy Films Evaporated at Grazing Incidence. I. Appl. Phvs. 1961, 32, S87.

(23) Smith, D. O.; Cohen, M. S.; Weiss, G. P. Oblique-Incidence Anisotropy in Evaporated Permalloy Films. I. Appl. Phvs. 1960, 31, $1755-1762$.

(24) Knorr, T. G.; Hoffman, R. W. Dependence of Geometric Magnetic Anisotropy in Thin Iron Films. Phvs. Rev. 1959, 113, 10391046.

(25) Kamberský, V.; Málek, Z.; Frait, Z.; Ondris, M. The dependence of the uniaxial magnetic anisotropy in evaporated films on the angle of incidence. Czech. I. Phvs. 1961, 11, 171-178.

(26) Raabe, J.; Tzvetkov, G.; Flechsig, U.; Böge, M.; Jaggi, A.; Sarafimov, B.; Vernooij, M. G. C.; Huthwelker, T.; Ade, H.; Kilcoyne, D.; Tyliszczak, T.; Fink, R. H.; Quitmann, C. PolLux: A new facility for soft $\mathrm{x}$-ray spectromicroscopy at the Swiss Light Source. Rev. Sci. Instrum. 2008, 79, 113704.

(27) Kimling, J.; Kronast, F.; Martens, S.; Böhnert, T.; Martens, M.; Herrero-Albillos, J.; Tati-Bismaths, L.; Merkt, U.; Nielsch, K.; Meier, G. Photoemission electron microscopy of three-dimensional magnetization configurations in core-shell nanostructures. Phvs. Rev. B: Condens. Matter Mater. Phys. 2011, 84, 174406.

(28) Streubel, R.; Kronast, F.; Fischer, P.; Parkinson, D.; Schmidt, O. G.; Makarov, D. Retrieving spin textures on curved magnetic thin films with full-field soft X-ray microscopies. Nat. Commun. 2015, 6, 7612. 